\title{
Detection of Sum and Difference Squeezing
}

\author{
Ranjana Prakash ${ }^{1}$ and Pramila Shukla ${ }^{1,2}$ * \\ ${ }^{l}$ Department of Physics, University of Allahabad, Allahabad, (India). \\ ${ }^{2}$ AIAS, AMITY University, Noida, (India).
}

\begin{abstract}
Sum and difference squeezing was defined by Hillery who showed that these turn into normal squeezing in sum and difference frequency generation. We re-examine this using an intense coherent pump mode and with a much better approximation. Our results are valid for much larger interaction times and therefore enable detection of smaller sum and difference squeezings, in principle., Moreover, if both, our and Hillary's results are regarded holding, our results lead to more squeezing.
\end{abstract}

Keywords: Coherent state; squeezing; sum and difference squeezing.

PACS Code: 42.50.Dv; 42.50.Ar; 42.50.-p

\section{Introduction}

Nonclassiclal phenomenon have important role in Quantum optics. Squeezing [1], antibunching [2], collapses- revivals [3] and quantum beats [4] were the earliest studied nonclassical phenomenon. There are some recent developments in higher order nonclassical states [5]. Earlier study of such non-classical phenomenon was largely in academic interest [6]. Now Squeezing has attracted considerable attention owing to application in the reducing noise in a signal [7], resonance fluorescence [8], detection of very weak forces such as gravitational waves [9], quantum teleportation [10] and others. Squeezing has been studied by some nonlinear optical process e.g. parametric amplification [11], multiwave mixing process [12-14], harmonic generation [15], Jaynes Cummings Model [16], and others.

Different type of squeezing was studied by authors [17-26]. Higher order squeezing in a single mode has been proposed by several authors. For a single mode this is introduced by Hong and Mandel [18] and by Hillery [19]. Hillery formulation was used to investigate the amplitude squared squeezing in spontaneous degenerate hyper Raman process [20]. Hillery also proposed [21] sum and difference squeezing for two-mode light. This was generalized to three modes [22, 23] and to even an arbitrary number of modes [24, 25]. Hillery [21] showed that detection of these are possible, in principle, using a process with a trilinear Hamiltonian involving three modes. Among these three modes two are those whose sum or difference squeezing is to be determined and the third is a pump mode. Perturbation expansions were used and it was shown that the ordinary squeezing of the pump mode after a nonzero interaction time gives sum or difference squeezing of the two input mode, i.e., the sum or difference squeezing before enter into the interaction described by the trilinear Hamiltonian. Giri and Gupta [26] reported the same for a Raman Process. They also discussed [26] sum and difference squeezing of the two modes after interaction for some time.

Detection of sum and difference squeezings of two modes by coupling them to an intense pump mode and then by detecting ordinary squeezing of the pump mode has been discussed by Hillery [19] and by Giri and Gupta [26].

In the present paper, we reexamine the sum and difference squeezings using an intense coherent pump mode with a much better approximation and our results are valid for larger interaction times so that even smaller sum and difference squeezing are detectable in principle. We make perturbation expansions in powers of reciprocal of the amplitude of intense pump mode and not in powers of coupling time as is done by others [21$23,26]$. Not only our results are valid for longer time intervals, at times, when our results are valid and Hillary's results are not valid, our results are seen to give a much larger squeezing then Hillary's.

\section{Sum squeezing of the two modes in terms of ordinary squeezing of an intense pump} mode in three wave interaction

Sum frequency of two modes, say, modes $B$ and $C$ having annihilation operator $b, c$ and creation operators $b^{\dagger}, c^{\dagger}$, is defined in terms of operators $W_{1,2}$ defined by

which obey

$$
W_{1}+\mathrm{i} W_{2} \equiv b c,
$$

$$
\left[W_{1}, W_{2}\right]=\mathrm{i} \frac{1}{2}\left(N_{b}+N_{c}+1\right), \Delta W_{1}^{2} \Delta W_{2}^{2} \geq \frac{1}{16}\left\langle N_{b}+N_{c}+1\right\rangle^{2} .
$$


A state is said to be sum squeezed in $W_{1}$ or in $W_{2}$ according as $\left(\Delta W_{1}\right)^{2}$ or $\left(\Delta W_{2}\right)^{2}$ is $<\frac{1}{4}\left\langle N_{b}+N_{c}+1\right\rangle$.

To measure sum squeezing of modes $B$ and $C$, we couple them to an intense pump mode $A$, which has operators $a$ and $a^{\dagger}$ with a sum-frequency resonance, i.e., $\omega_{A}=\omega_{B}+\omega_{C}$. The 3-wave interaction is described by the interaction picture Hamiltonian,

$$
H_{\mathrm{I}}=g\left(a^{\dagger} b c+a b^{\dagger} c^{\dagger}\right) \text {, }
$$

where $a^{\dagger}(a), b^{\dagger}(b)$ and $c^{\dagger}(c)$ are the interaction picture creation (annihilation) operators of the $A$, $B$ and $C$ modes respectively and $g$ is the coupling constant of the interaction. We consider an intense pump mode initially in the coherent state $\left|x e^{i \theta}\right\rangle$ with $x>>1$, and write $a=\left(x+A_{\theta}\right) e^{i \theta}$.

The interaction Hamiltonian then, can, be written in the form,

$$
H_{\mathrm{I}}=G\left[\left(B C+B^{\dagger} C^{\dagger}\right)\right]+\frac{1}{x} G\left[A_{\theta}^{\dagger} B C+A_{\theta} B^{\dagger} C^{\dagger}\right] \text {, }
$$

where operators $B, C$ and constant $G$ are defined by

$$
b=B e^{i \theta / 2}, c=C e^{i \theta / 2} \text { and } G=g x .
$$

It may be noted that, for $x>>1, G t$, may be appreciable even for a small value of $g t$. We shall do calculations exact in $G t$ but with a perturbation expansion in $1 / x$, correct to first order only. We write subscript $\theta$ on operator of $A$ mode as, later, we consider various values of $\theta$, the phase of coherent pump state. Although operators $B$ and $C$ also depends on $\theta$, we do not put a subscript on them as they do not appear in final results.

Equations of motion for operators $A_{\theta}, B, C$ are

$$
\dot{A}_{\theta}=-\mathrm{i} \frac{1}{x} G B C, \quad \dot{B}=-\mathrm{i} G C^{\dagger}-\mathrm{i} \frac{1}{x} G A C^{\dagger}, \quad \dot{C}=-\mathrm{i} G B^{\dagger}-\mathrm{i} \frac{1}{x} G A B^{\dagger}
$$

These equations can be solved by perturbation to second order, the result for $\mathrm{A}_{\theta}$ is

$$
\begin{aligned}
& A_{\theta}(t)=A_{\theta S}+\frac{1}{4 x}\left[-\left(B_{S}^{\dagger} B_{S}+C_{S}^{\dagger} C_{S}+1\right)(\cosh 2 \mathrm{G} \mathrm{t}-1)-\mathrm{i}\left(B_{S} C_{S}-B_{S}^{\dagger} C_{S}^{\dagger}\right) \sinh 2 \mathrm{G} \mathrm{t}\right. \\
& \left.-2 \mathrm{iGt}\left(B_{S} C_{S}+\mathrm{B}_{S}^{\dagger} C_{S}^{\dagger}\right)\right] \\
& -\frac{1}{8 \mathrm{x}^{2}}\left(A_{\theta S}+A_{\theta S^{\dagger}}{ }^{\dagger}\right)\left[\left(B_{S}^{\dagger} B_{S}+C_{S}^{\dagger} C_{S}+1\right)(2 \mathrm{Gt} \sinh 2 \mathrm{Gt}-\cosh 2 \mathrm{Gt}+1)\right. \\
& \left.+\mathrm{i}\left(B_{S} C_{S}-B_{S}{ }^{\dagger} C_{S}^{\dagger}\right)(2 \mathrm{Gt} \cosh 2 \mathrm{Gt}-\sinh 2 \mathrm{G} \mathrm{t})\right] \\
& +\frac{1}{8 \mathrm{x}^{2}}\left(A_{\theta S}{ }^{\dagger}-A_{\theta S}\right)\left[\left(B_{S}^{\dagger} B_{S}+C_{S}^{\dagger} C_{S}+1\right)(\cosh 2 \mathrm{G} \mathrm{t}-1)-2 \mathrm{i} B_{S}{ }^{\dagger} \mathrm{C}_{S}^{\dagger} \sinh 2 \mathrm{G} \mathrm{t}\right. \\
& \left.+4 \text { i G t } B_{S}^{\dagger} C_{S}^{\dagger}\right]
\end{aligned}
$$

Here, suffix $S$ on an operator refers to Schrodinger picture which gives value of the operator at $t=0$.

To examine the squeezing of the field amplitude of the pump mode $A$, we define general operator

$$
X_{\theta \varphi}(t)=\frac{1}{2}\left[A_{\theta} e^{-i \varphi}+A_{\theta}^{\dagger} e^{i \varphi}\right] \text {. }
$$

Using above equation we obtain

$$
\begin{aligned}
& X_{\theta \varphi}(t)=X_{\theta \varphi S}+\frac{1}{2 x}[-W_{3} f_{1} \cos \varphi+\left(-\mathrm{W}_{1} \sin \theta+W_{2} \cos \theta\right) f_{2} \cos \varphi \\
&\left.-\left(W_{1} \cos \theta+W_{2} \sin \theta\right) f_{3} \sin \varphi\right] \\
&-\frac{1}{2 x^{2}} \mathrm{X}_{\theta 0 S}\left[W_{3}\left(f_{2} f_{3}-f_{1}\right) \cos \varphi+\left(W_{1} \sin \theta-W_{2} \cos \theta\right)\left(f_{1} f_{3}+f_{3}-f_{2}\right) \cos \varphi\right] \\
&-\frac{1}{2 x^{2}} \mathrm{X}_{\theta \frac{\pi}{2} S}\left[W_{3} f_{1} \sin \varphi-\left\{W_{1} \cos (\theta-\varphi)+W_{2} \sin (\theta-\varphi)\right\}\left(f_{2}-f_{3}\right)\right],
\end{aligned}
$$

where $\quad X_{\theta \varphi S}=\frac{1}{2}\left(A_{\theta S} e^{-i \varphi}+A_{\theta S} e^{i \varphi}\right)$, and $f_{1,2,3}$ are defined by $f_{1}=(\cosh 2 G t-1)$, $f_{2}=\sinh 2 G t, f_{3}=2 G t$ and $W_{3}=\frac{1}{2}\left(N_{b}+N_{c}+1\right)$.

It may be noted that for $\mathrm{Gt}<<1, f_{1} \approx 2 G^{2} t^{2}, f_{2}-f_{3} \approx \frac{4}{3} G^{3} t^{3}$ and $\Delta X_{\theta \frac{\pi}{2}}^{2}(t)-\frac{1}{4} \propto t^{2}$. 
The variance of $X_{\theta \frac{\pi}{2}}(t)$ is obtained as

$$
\begin{aligned}
\Delta X_{\theta \frac{\pi}{2}}^{2}(t)=\frac{1}{4}+\frac{1}{4 x^{2}}[ & \Delta W_{1}^{2} \cos ^{2} \theta+\Delta W_{2}^{2} \sin ^{2} \theta+\left(\left\langle W_{1} W_{2}\right\rangle+\left\langle W_{2} W_{1}\right\rangle\right. \\
& \left.\left.-2\left\langle W_{1}\right\rangle\left\langle W_{2}\right\rangle\right) \sin \theta \cos \theta\right] \mathrm{f}_{3}{ }^{2} \\
& -\frac{1}{4 x^{2}}\left[\left\langle W_{3}\right\rangle f_{1}-\left(f_{2}-f_{3}\right)\left\langle W_{1} \sin \theta-W_{2} \cos \theta\right\rangle\right] .
\end{aligned}
$$

The above equation can be solved for $\left(\Delta W_{1}\right)^{2}$ and $\left(\Delta W_{2}\right)^{2}$ and gives

$$
\begin{aligned}
& \left(\Delta W_{1}\right)^{2}=\frac{x^{2}}{2 G^{2} t^{2}}\left[\Delta X_{0 \frac{\pi}{2}}^{2}+\Delta X_{\pi \frac{\pi}{2}}^{2}-\frac{1}{2}\right]+\frac{1}{8 G^{2} t^{2}}\left\langle N_{b}+N_{c}+1\right\rangle(\cosh 2 G t-1), \\
& \left(\Delta W_{2}\right)^{2}=\frac{x^{2}}{2 G^{2} t^{2}}\left[\Delta X_{\frac{\pi}{2} \frac{\pi}{2}}^{2}+\Delta X_{\frac{3 \pi}{2} \frac{\pi}{2}}^{2}-\frac{1}{2}\right]+\frac{1}{8 G^{2} t^{2}}\left\langle N_{b}+N_{c}+1\right\rangle(\cosh 2 G t-1) .
\end{aligned}
$$

These equations give us the relation between sum squeezing of the two given input modes in terms of normal squeezing of the intense pump modes after 3-wave interaction for some time, for a fixed $\varphi$ and different $\theta$.

\section{Difference squeezing of the two modes in terms of ordinary squeezing of an intense pump mode in three wave interaction}

Difference frequency of two modes, say, modes $B$ and $C$ having annihilation operator $b, c$ and creation operators $b^{\dagger}, c^{\dagger}$ is defined in terms of operators $V_{1,2}$ defined by

which obey

$$
V_{1}+i V_{2} \equiv b^{\dagger} c
$$

$$
\left[V_{1}, V_{2}\right]=i \frac{1}{2}\left(N_{b}-N_{c}\right), \quad \Delta V_{1}^{2} \Delta V_{2}^{2} \geq \frac{1}{16}\left\langle N_{b}-N_{c}\right\rangle^{2} .
$$

A state is said to be difference squeezed in $V_{1}$ or in $V_{2}$ according as $\left(\Delta V_{1}\right)^{2}$ or $\left(\Delta V_{2}\right)^{2}$ is $<\frac{1}{4}\left\langle N_{b}-N_{c}\right\rangle$.

To measure difference squeezing of modes $B$ and $C$, we couple them to an intense pump mode A which has operators $a$ and $a^{\dagger}$ with a difference frequency resonance $\omega_{A}=\omega_{C}-\omega_{B}$. Note that we call that input mode as $C$ which has larger frequency without loss of generality. The 3-wave interaction is described by the interaction picture Hamiltonian,

$$
H_{\mathrm{I}}=g\left(a^{\dagger} b^{\dagger} c+a b c^{\dagger}\right) \text {, }
$$

We write $a=\left(x+A_{\theta}\right) e^{i \theta}, b=B e^{-i \theta / 2}$ and $c=C e^{\mathrm{i} \theta / 2}$ and the interaction Hamiltonian takes the form,

$$
H_{\mathrm{I}}=G\left[\left(B^{\dagger} C+B C^{\dagger}\right)+\frac{G}{x}\left(A_{\theta}^{\dagger} B^{\dagger} C+A_{\theta} B C^{\dagger}\right)\right], \quad G=g x .
$$

We do calculations exact in $G t$ but with perturbation expansion in $1 / x$, correct to first order only exactly as we did for sum squeezing.

Equations of motion for operators $A_{\theta}, B, C$ are

$$
\dot{A} \theta=-\mathrm{i} \frac{1}{x} G B^{\dagger} C, \quad \dot{B}=-i G C-\mathrm{i} \frac{1}{x} G A^{\dagger} C^{\dagger}, \quad \dot{C}=-i G B-i \frac{1}{x} G A B,
$$

and they lead to

$$
\begin{gathered}
A_{\theta}(\mathrm{t})=A_{\theta S}+\frac{1}{4 x}\left[\left(B_{S}{ }^{\dagger} B_{S}-C_{S}^{\dagger} C_{S}\right)(\cos 2 G t-1)-\mathrm{i}\left(B_{S}{ }^{\dagger} C_{S}-B_{S} C_{S}^{\dagger}\right) \sin 2 G t\right. \\
\left.-2 \mathrm{iGt}\left(B_{S}^{\dagger} C_{S}+B_{S} C_{S}^{\dagger}\right)\right] \\
-\frac{1}{8 x^{2}}\left(A_{\theta S}+A_{\theta S}{ }^{\dagger}\right)\left[\left(B_{S}^{\dagger} B_{S}-C_{S}^{\dagger} C_{S}\right)(2 G t \sin 2 G t+\cos 2 G t-1)\right. \\
\left.+\mathrm{i}\left(B_{S}^{\dagger} C_{S}-B_{S} C_{S}^{\dagger}\right)(2 G t \cos 2 G t-\sin 2 G t)\right] \\
-\frac{1}{8 x^{2}}\left(A_{\theta S}^{\dagger}-A_{\theta S}\right)\left[\left(B_{S}^{\dagger} B_{S}-C_{S}^{\dagger} C_{S}\right)(\cos 2 G t-1)+2 \mathrm{i} B_{S} C_{S}^{\dagger} \sin 2 G t\right. \\
\left.-4 \mathrm{i} G t B_{S} C_{S}^{\dagger}\right] .
\end{gathered}
$$

Using equation (8) and (18) we obtain 


$$
\begin{aligned}
& X_{\theta \varphi}(\mathrm{t})= X_{\theta \varphi S}+\frac{1}{2 x}\left[V_{3} k_{1} \cos \varphi+\left(-V_{1} \sin \theta+V_{2} \cos \theta\right) k_{2} \cos \varphi\right. \\
&\left.-\left(V_{1} \cos \theta+V_{2} \sin \theta\right) k_{3} \sin \varphi\right] \\
&-\frac{1}{2 x^{2}} \mathrm{X}_{\theta 0 S}\left[V_{3}\left(k_{2} k_{3}+k_{1}\right) \cos \varphi+\left(V_{1} \sin \theta-V_{2} \cos \theta\right)\left(k_{1} k_{3}+k_{3}-k_{2}\right) \cos \varphi\right] \\
&-\frac{1}{2 x^{2}} \mathrm{X}_{\theta \frac{\pi}{2} S}\left[-V_{3} k_{1} \sin \varphi+\left\{V_{1} \cos (\theta-\varphi)+V_{2} \sin (\theta-\varphi)\right\}\left(k_{2}-k_{3}\right)\right] .
\end{aligned}
$$

where $k_{1,2,3}$ are defined by $k_{1}=(\cos 2 G t-1), \quad k_{2}=\sin 2 G t, \quad k_{3}=2 G t$ and

$V_{3}=\left(N_{b}-N_{c}\right) / 2$.

Here, we note that, for $\mathrm{Gt}<<1, k_{1} \approx 2 G^{2} t^{2}, k_{2}-k_{3} \approx \frac{4}{3} G^{3} t^{3}$ and $\Delta X_{\theta \frac{\pi}{2}}^{2}(t)-\frac{1}{4} \propto t^{2}$.

Variance of $X_{\theta \frac{\pi}{2}}(t)$ is obtained as

$$
\begin{aligned}
\Delta X_{\theta \frac{\pi}{2}}^{2}(t)=\frac{1}{4}+\frac{1}{4 x^{2}}[ & \Delta V_{1}^{2} \cos ^{2} \theta+\Delta V_{2}^{2} \sin ^{2} \theta+\left(\left\langle V_{1} V_{2}\right\rangle+\left\langle V_{2} V_{1}\right\rangle\right. \\
& \left.\left.-\left\langle V_{1}\right\rangle\left\langle V_{2}\right\rangle-\left\langle V_{2}\right\rangle\left\langle V_{1}\right\rangle\right) \sin \theta \cos \theta\right] k_{3}^{2} \\
- & \frac{1}{4 x^{2}}\left[-\left\langle V_{3}\right\rangle k_{1}+\left(k_{2}-k_{3}\right)\left\langle V_{1} \sin \theta-V_{2} \cos \theta\right\rangle\right] .
\end{aligned}
$$

The above equation can be solved for $\left(\Delta \mathrm{V}_{1}\right)^{2}$ and $\left(\Delta \mathrm{V}_{2}\right)^{2}$, and gives

$$
\begin{aligned}
& \left(\Delta \mathrm{V}_{1}\right)^{2}=\frac{\mathrm{x}^{2}}{2 \mathrm{G}^{2} \mathrm{t}^{2}}\left[\Delta \mathrm{X}_{0 \frac{\pi}{2}}^{2}+\Delta \mathrm{X}_{\pi \frac{\pi}{2}}^{2}-\frac{1}{2}\right]-\frac{1}{8 \mathrm{G}^{2} \mathrm{t}^{2}}\left\langle\mathrm{~N}_{\mathrm{b}}-\mathrm{N}_{\mathrm{c}}\right\rangle(\cos 2 \mathrm{Gt}-1), \\
& \left(\Delta \mathrm{V}_{2}\right)^{2}=\frac{\mathrm{x}^{2}}{2 \mathrm{G}^{2} \mathrm{t}^{2}}\left[\Delta \mathrm{X}^{2}{ }_{\frac{\pi}{2} \frac{\pi}{2}}+\Delta \mathrm{X}_{\frac{3 \pi}{2} \frac{\pi}{2}}^{2}-\frac{1}{2}\right]-\frac{1}{8 \mathrm{G}^{2} \mathrm{t}^{2}}\left\langle\mathrm{~N}_{\mathrm{b}}-\mathrm{N}_{\mathrm{c}}\right\rangle(\cos 2 \mathrm{Gt}-1) .
\end{aligned}
$$

These equations give us the relation between difference squeezing of the two given input modes in terms of normal squeezing of the intense pump modes after 3-wave interaction for some time, for a fixed $\phi$ and different $\theta$.

\section{Discussion, Conclusions and suggestion for experimental verification}

Validity of first order perturbation theory results may be represented by $\left\langle\left[\int H_{\mathrm{I}}(t) d t\right]\right\rangle<<1$. For Hillery's case it gives gt $<1 / \sqrt{n_{a} n_{b} n_{c}}$ which is equivalent to Gt $<1 / \sqrt{n_{b} n_{c}}$. Here, $n_{a}, n_{b}, n_{c}$ are the number of photons in the pump and the two given modes. Our results for sum squeezing are however seen to be valid for $\operatorname{Sinh} 2 \mathrm{Gt}<\sqrt{n_{a} / n_{b} n_{c}}$ by replacing $H_{\mathrm{I}}$ above by the part of $H_{\mathrm{I}}$ in which we make first order perturbation expansion. Our results obviously hold for much larger durations as compared to Hillary's results. We plot a graph with Gt vs $\Delta X_{\theta \frac{\pi}{2}}^{2}(t)-\frac{1}{4}$ for sum squeezing for $\theta=0$ and $\pi$ for some typical values of $\Delta W_{1}^{2}, \Delta W_{2}^{2},\left\langle W_{1} W_{2}\right\rangle,\left\langle W_{1}\right\rangle,\left\langle W_{2}\right\rangle, N_{b}, N_{c}, \Delta V_{1}^{2}, \Delta V_{2}^{2},\left\langle V_{1} V_{2}\right\rangle,\left\langle V_{1}\right\rangle,\left\langle V_{2}\right\rangle$ and also show Hillery's result in Figure (1). It shows that our results bring much larger squeezing. We compare these graphical results with Hillery's results.

Similarly, for difference squeezing our results are valid for $2 \mathrm{Gt}<<\sqrt{n_{a} / n_{b} n_{c}}$. Then thus hold for much larger duration than the Hillary's results which holds for $\mathrm{Gt}<<1 / \sqrt{n_{b} n_{c}}$. In Figure (2), we show variation of our results for $\theta=0$ and $\pi$ and the Hillery's results for comparison. Our results give larger squeezing.

We note that our results for both sum and difference squeezing give larger squeezing and hold for larger duration as compared to Hillery's results. These should, therefore, enable detection of smaller sum and difference squeezing, in principle

Since sum and difference squeezing of modes $B$ and $C$ is connected to ordinary squeezing of intense pump mode, it can be detected using the usual homodyning method [27]. The sum (or difference) squeezed light 
may be mixed by a beam splitter with the coherent light of a strong local pump oscillator, whose phase $\theta$ can be varied by a delay line used before mixing with input modes.

\section{Acknowledgement}

We are thankful to Prof. Hari Prakash and Prof. Naresh Chandra for their interest and critical comments and for fruitful discussions.

\section{References}

[1]. $\quad$ D F Walls Nature 306141 (1983); R Loudon \& P L Knight J Mod Opt 34709 (1987).

[2]. N Chandra and H Prakash Phys Rev A 11696 (1970); H Paul Rev. Mod. Phys 541061 (1982).

[3]. Ranjana Prakash and Pramila Shukla International Journal of Pure and Applied Physics 222463 (2008)

[4]. Ranjana Prakash and Naresh Chandra Phys. Rev. Lett. 42443 (1979).

[5]. Anirban Pathak and Amit Verma Indian J. Phys. 841005 (2010).

[6]. B R Mollow and R J Glauber Phys Rev 1601076 (1967).

[7]. H P Yuen Phys Rev A 132226 (1976).

[8]. D F Walls and P Zoller Phys Rev Lett 47709 (1981).

[9]. R Loudon Phys Rev Lett 47815 (1981).

[10]. S L Braunstein and H J Kimble Phys Rev Lett 80869 (1998).

[11]. L A Wu, H J Kimble, J L Hall \& H Wu Phys Rev Lett, 572520 (1986).

[12]. M D Reid \& D F Walls Phys Rev A 311622 (1985).

[13]. P Marian Phys Rev A 443325 (1991).

[14]. S. Rani, J. Lal and N. Singh Indian J. Phys. 8653 (2012).

[15]. L Mandel, Opt Comm 42437 (1982).

[16]. M H Mahran and A S F Obada Phys Rev A 404476 (1989).

[17]. Ranjana Prakash and Namrata Shukla, Optics Communications 2843568 (2011).

[18]. K Kong \& L Mandel Phys Rev A 32974 (1985).

[19]. M Hillery Opt Commn 62135 (1987); M Hillery Phys Rev 363796 (1987).

[20]. Biswajit Sen and Swapan Mandal Indian J. Phys.84 1111 (2010).

[21]. M Hillery Phys Rev A 403147 (1989).

[22]. Kumar and P S Gupta Opt Commn 136441 (1997).

[23]. Kumar and P S Gupta Quant Semiclass Opt 10485 (1998).

[24]. Ba An Nguyen and Vo Tinh Phys Lett A 26134 (1999).

[25]. Ba An Nguyen and Vo Tinh Phys Lett A 270 (2000) 27; Ba An Nguyen and Vo Tinh J Phys A 332951 (2000).

[26]. D K Giri and P S Gupta Modern Phys Lett B 191261 (2005).

[27]. L Mandel Phys Rev Lett 49136 (1982).

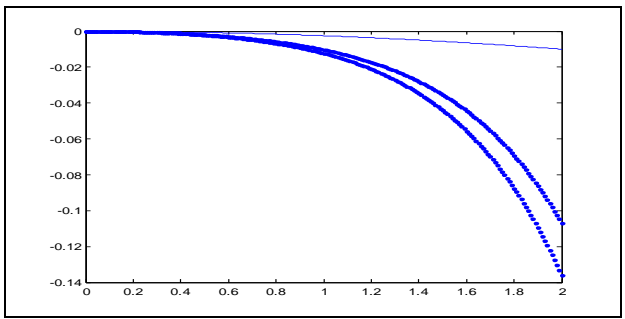

Figure1. Graph showing variation of $\Delta X_{\theta \frac{\pi}{2}}^{2}(t)-\frac{1}{4}$ with Gt in case of sum squeezing. $\Delta W_{1}^{2}=\Delta W_{2}^{2}=\left\langle W_{1} W_{2}\right\rangle=\left\langle W_{1}\right\rangle=\left\langle W_{2}\right\rangle=1 / 4, N_{b}=N_{c}=1 / 2$. Dotted line shows our result (upper for $\theta=0$ and lower for $\theta=\pi)$ and solid line shows Giri and Gupta's result.

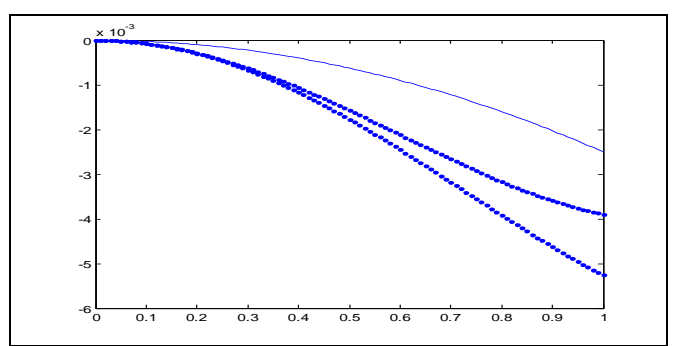

Figure2. Graph showing variation of $\Delta X_{\theta \frac{\pi}{2}}^{2}(t)-\frac{1}{4}$ with Gt in case of difference squeezing. $\Delta \mathrm{V}_{1}^{2}=\Delta \mathrm{V}_{2}^{2}=\left\langle V_{1} V_{2}\right\rangle=\left\langle V_{1}\right\rangle=\left\langle\mathrm{V}_{2}\right\rangle=1 / 4, N_{b}=4, N_{c}=2$. Dotted line shows our result (upper for $\theta=0$ and lower for $\theta=\pi$ ) and solid line shows Giri and Gupta's result. 